\title{
The role of passive defense in reducing the risks of earthquakes
}

\author{
Mohammadreza Nikoumanesh ${ }^{1, *}$, Alireza Nazarkhah ${ }^{2}$ \\ ${ }^{1}$ Civil Engineering department, Islamic Azad University, Central Tehran Branch, Tehran, Iran \\ ${ }^{2}$ Industrial Engineering department, Islamic Azad University, Central Tehran Branch, Tehran, Iran
}

\section{Email address:}

mim_nikoo@yahoo.com (M. Nikoumanesh),nazarqa@yahoo.com (A. Nazarkhah)

\section{To cite this article:}

Mohammadreza Nikoumanesh, Alireza Nazarkhah. The Role of Passive Defense in Reducing the Risks of Earthquakes. American Journal of Civil Engineering. Special Issue: Research and Practices of Civil Engineering in Developing Countries. Vol. 3, No. 2-2, 2015 , pp. 6-9. doi: $10.11648 /$ j.ajce.s.2015030202.12

\begin{abstract}
The protection and stabilization of populous industrial cities that are near active faults in Iran against risks of earthquakes are of important duties of each Iranian. The goals of passive defense against earthquakes include the continuation of infrastructure activities, provision of essential requirements, continuity of public services, and facilitation of the country's management in threatening conditions, crises, earthquakes, and any other natural events and disasters. Maintenance of the government's managing power is possible through the implementation of passive defense projects and reduction of the vulnerability of vital structures. Therefore, every program designed and implemented as the passive defense seeks to increase the capacity of the country for dealing with probable threats of any natural disasters, especially earthquakes, and also improve the public tolerance threshold and capabilities to face with emergencies and crises besides paying attention to security and economic problems.
\end{abstract}

Keywords: Earthquakes, Passive Defense, Risk Reduction

\section{Introduction}

The reduction of risks and damages of earthquakes has always received the attention of managers, researchers, and the public. Any activity about earthquakes, either before or after them, which is performed to increase safety against earthquakes is considered important. The history of countries pioneer and successful in this regard shows the importance and applicability of such activities [1]. Dealing with any risk and adversity is usually called defense. Today, defense is defined and used as active and passive. Active defense is usually important in military activities, based on activities of armed forces, and relied on military weapons and equipment that repel the attacks and neutralize hostile actions.

On the contrary, passive defense refers to a type of defense that does not rely on military weapons and can prevent the financial damage and life losses. More to the point, any nonarmed action that reduces the vulnerability of human resources, buildings, installations, equipment, documents, and lifelines of the country against enemies' attacks and natural disasters is called passive defense.

The passive defense methods involve a broad spectrum of political, economical, social, and construction actions. Now, most countries try to design and implement strategic and integrated plans for the passive defense through a regular schedule. These countries use logistic models for settlement of people, installations and equipment required for retrofitting the buildings, multipurpose use of existing spaces in construction projects, and designing and implementing the operational programs for crisis management.

\section{The Objective of the Passive Defense against Earthquakes}

Iran has large industrial cities near the active faults, and protection and stabilization of these cities are of important duties of every Iranian. The objectives of passive defense against earthquakes include the continuation of infrastructure activities, provision of essential needs, continuity of public services, and facilitation of the country's management during and even before threatening conditions, crises, and 
earthquakes. In this regard, maintenance of the government's managing power is possible through implementing passive defense projects and reducing vulnerability of vital structures. Therefore, every program designed and implemented as passive defense tries to increase the capacity of the country for dealing with probable threats of any natural disasters, especially earthquakes, and also improve the public tolerance threshold and capabilities to face with emergencies and crises besides paying attention to security and economic problems.

The capabilities of passive defense that should be usually established against any military threat or natural threats, including earthquakes, floods, tsunamis, and other disasters, are as follows:

- Making a suitable context for sustainable development of the country;

- Complying with de-stressing policies;

- The most stable and cost-effective method of dealing with natural risks and disasters;

- The most appropriate strategy for increasing the national tolerance threshold;

- Supporting the national authority;

- The most favorable and appropriate method for reducing the risks and vulnerability; and

- The most intrinsic defensive component of humans against all events.

In addition to the above items, passive defense against earthquakes is a dynamic component, so that, it can be a priority for scientific and research efforts and be taught and developed in all managing engineering levels [2].

If passive defense is not considered in dealing with earthquakes, the following risks, damages, and problems may incur the country:

- Damage or destruction of buildings and infrastructures;

- Interrupted or impaired provision of services following the power, gas, and water cut;

- Dysfunction of computerized systems and telecommunication networks;

- Pollution and outbreak of diseases due to the corpses and sewage fallen to the surface and underground water and shortage of fresh potable water;

- Incidence of secondary events following gas leakage and power transmission line defects;

- Disrupted provision of disaster relief services to injured people due to the closure of urban accesses; and

- Problems of accommodation and preparation and distribution of primary needs of the injured people.

The immunization of vital, critical, and important centers should receive attention in order to reach the above strategic objectives and solve the expected and unexpected problems. The important centers regarding passive defense are as follows:

Vital centers: These centers involve the national activity expansion; the establishment and continuation of their activities are vital for the country, and damages to these centers disturb the control of government's affairs.

Critical centers: These centers involve the regional activity expansion; the establishment and continuation of their activities are essential for some regions of the country, and damages to these centers result in dysfunction of a part of the country.

Important centers: These centers involve the regional activity expansion; the establishment and continuation of their activities are important for a part of country, and damages to these centers disturb that part of country.

The promotion of survival and maintenance of the country in crises resulting from earthquakes in large cities is another objective of passive defense.

The culturalization and development of public belief about the effectiveness of passive defense in reducing vulnerability, especially in educational centers, such as schools, universities, and governmental departments, is a very essential step that reduces the crisis and its consequences. The earthquake drills performed in schools and the resulting inductive culture transferred to students' homes over recent years are evidence of the above-mentioned step. The fundamental objectives in this regard are as follows:

- Attainment of sustainable security in development and stabilization of vital infrastructures of the country;

- Performing research, producing knowledge and technology, culturalizing, and converting the knowledge to information and public awareness;

- Institutionalizing the respect for principles and standards of passive defense in development projects;

- Reducing the vulnerable parts of the country and revealing the resulting national authority as the promotion of knowledge and dealing with potential and actual risks of earthquakes;

- Minimizing the effect of earthquake threats on vital, critical, and important infrastructures; and

- Developing the executive capacity of the passive defense in engineering context of the country qualitatively and quantitatively (consultancy and execution).

\section{Principles, Fundamental Subjects, and Strategies of the Passive Defense against Earthquakes}

To achieve the objectives mentioned in the previous section, the principles and strategies of passive defense should receive attention. The principles of passive defense cannot be separated from macro-policies of the country; rather, they should be approved and implemented within short-term and long-term programs. The subjects and strategies of passive defense can be generally discussed in development of public culture, promotion of technology, optimization of industrial production in all technical areas of the country, and following items:

- Extensive interaction with organizations and establishing appropriate mechanisms for immunizing and protecting the infrastructure installations;

- Playing the instructive and supervising role for relevant organizations and institutions in terms of studying and 
technical designing of passive defense projects;

- Utilizing all facilities for making systems and methods of passive defense cheap, diverse, and creative;

- Effective development and interaction with policymaking, legislative, and administrative bodies of the country;

- Scientific development, technical knowledge production, promotion of technology and comprehensive educational programs, and optimization of the industrial production using all capacities;

- Institutionalizing the comprehensive system and using the principles and standards in development projects of the country; and

- Developing the culture and institutionalizing the public belief about the effectiveness of passive defense in reducing vulnerability.

Different factors should be considered to define the principles and fundamental subjects of passive defense against earthquakes. The desired objectives can be reached faster, better, and more easily, and risks of earthquakes can be reduced when complying with the following items:

- Locating and establishing the functions

Choosing an appropriate place which has the capacity for accomplishment of the relevant activity and suitable conditions for future developments in a way that the principles of passive defense are observed besides the general factors.

- Constructing the vital, critical, and important centers in places that receive minimum damages when earthquakes occur and are easily accessible after earthquakes.

- Dispersion

The dispersion of fundamental components is an important subject of passive defense and is essential in reducing damages caused by natural disasters. Construction of huge industrial complexes, large power plants, and similar items in the same area of the country is not reasonable; rather, they had better be constructed in different parts of the country respecting the principles of dispersion and appropriate for geography of the country.

- Training and culturalizing

Authorities, decision makers, and all people who somehow contribute to policy-making of preparation and implementation of passive defense projects should provide general instructions to people, when necessary, through the media and try to culturalize how to deal with risks of earthquakes through distributing brochures and providing practical drills.

- Access ways

Access ways refer to passage and communicative roads and areas and can be planned in different types and scales. The communication lines of a place to the outside environment should be based on standards in a way that the threats of earthquakes do not disturb or stop the traffic.

- Determining the optimum scale for settlement of people

- Downsizing the residential units and making them at lower costs
- Localization

- Quantitative and qualitative development of professional human resources

- The capacity to be repaired and reconstructed

- Retrofitting

The retrofitting of existing buildings is an important item for passive defense. The destructions incurred by large earthquakes in Iran show the unfavorable quality of constructions. Old buildings constructed before the development of the Codes 2800 and its amendments show fundamental problems during earthquakes, so that, they should be retrofitted.

Most of the Iranian cities near the fault lines are old and located in distressed areas with narrow alleys and streets, and thus, provision of services after earthquakes is very difficult and limited. Most of them cannot be repaired or retrofitted and should be reconstructed. Although the governmental banks offer low-interest loans for reconstruction of buildings in distressed areas, the construction in these areas progresses very slowly due to the lack of vital lifelines, small size of existing passages, and other factors.

Old buildings often have load-bearing walls or a steelskeleton frame that are not much resistant against lateral loads and do not have any cross bracing system or component resisting against earthquakes.

The schools located in such areas should be also repaired, retrofitted, and remedied. In this regard, the School Renovation Organization has begun valuable efforts to retrofit the educational buildings and schools, which should be continued more rapidly.

A suggested, passive defense for the distressed areas is the safe room plan. The old buildings in distressed areas generally have load-bearing walls without vertical and horizontal ties and are very likely to be destroyed by earthquakes. In the safe room plan, a certain part of the building where the residents are present at the time of earthquakes is immunized through constructing a strong structure. The structure prevents the fall of debris into the safe room when an earthquake occurs and the building is getting destroyed and actually makes a protective area to save the residents, which is the major role of the passive defense. It is better to allocate one or two rooms where the family members are mostly present to safe room plan. Obviously, there is a few vital seconds for transfer of the residents from different parts of the building to the safe room at the time of earthquakes, from the beginning of vibration to the destruction of the building.

In the safe room plan, a metal frame is embedded in each floor of the building in order that the debris cannot be fallen on the residents during the earthquake and after collapse of the building. Such a room can be made for buildings under construction.

Advantages of safe rooms include the capability to hide the frame using various architectural models, low cost (about 600 USD), considerable reduction of risks and human casualties, high speed of construction, and the capability to be adapted with various types of structural systems. The size 
of safe rooms is $3 \times 4 \mathrm{~m}^{2}$, and their columns are better to be aligned at different floors of the building.

The passive defense strategies against earthquakes are obvious in macro-planning of Iran as appropriate. The clauses of the draft general policies for the passive defense that has been examined by the Expediency Discernment Council of the System reveal the following important macro plans of Iran:

- Public culturalization on the necessity for the use of principles of the passive defense at governmental levels and expansion and enhancement of research and educational centers in order to produce and promote the technical knowledge in this regard;

Determining an entity for designing, planning, codifying, and ratifying the standards,

- The passive defense and controlling its activity in logistic, developmental, and industrial projects;

- Providing the necessary support for relevant industries for supplying professional equipment for the advanced and effective passive defense required for the country taking into account the use of domestic design and production;

- Classifying and prioritizing the vital centers and installations, prioritizing the passive defense, predicting necessary guarantees for protecting the classified information of these centers, and actions taken for their passive defense;

- Preventing the construction of dangerous installations in populated places and gradually transferring these buildings outside the city in order to reduce their additional risks; and

- Constructing dual-purpose buildings, installations and networks at present and in the future in order to exploit the conditions when the threats occur.

\section{Conclusion}

To deal with risks of earthquakes, the relevant passive defense should be established and emphasized by all people. In this regard, the following items should be respected:

1 An example of passive defense is to retrofit the buildings that were not constructed according to the requirements of constructional national regulations and the codes of earthquake protection.

2 Immunizing the vital, critical, and important centers

3 Culturalizing and developing the public belief

4 Performing the earthquake drills in schools and transferring the resulting inductive culture to students' homes

5 Constricting the vital, critical, and important centers in places that receive minimum damages when earthquakes occur and are easy accessible after earthquakes.

6 Dispersing the fundamental components, such as huge industrial complexes, large power plants, and similar items

7 Developing the human resources qualitatively and quantitatively

8 Designing and constructing safety rooms and similar cases

\section{References}

[1] H. Beirami Fam Maleki, "The role of the passive defense in management of the crisis caused by earthquakes" The first international conference of seismic retrofitting. Tabriz, Iran. Oct. $20^{\text {th }}-22^{\text {nd }}, 2008$.

[2] M. S. Jebel Amoli, A. Mirmohammad Sadeghi, Value engineering. Forat Publications, 2001. 